\title{
Triterpenoids and Sterones from the Stem Bark of Ailanthus altissima
}

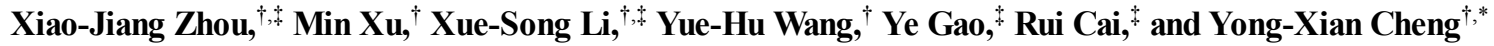 \\ ${ }^{\dagger}$ State Key Laboratory of Phytochemistry and Plant Resources in West China, Kunming Institute of Botany, \\ Chinese Academy of Sciences, Kunming 650204, China. E-mail: yxcheng@mail.kib.ac.cn \\ ${ }^{\ddagger}$ College of Pharmacy, Hunan University of Chinese Medicine, Changsha 410208, China
} Received July 28, 2010, Accepted November 2, 2010

\begin{abstract}
One new tirucallane-type triterpenoid, alianthusaltinin A (1), one new $\mathrm{C}_{29}$ sterone, alianthaltone A (2), and 12 known compounds have been isolated from the stem bark of Ailanthus altissima. The structures of new compounds were identified by means of spectroscopic methods. Compound $\mathbf{3}$ was isolated from natural sources for the first time, and compounds $\mathbf{4}, \mathbf{5}$, and $\mathbf{9}$ were isolated from this plant for the first time.
\end{abstract}

Key Words: Ailanthus altissima (Simaroubaceae), Tirucallane-type triterpenoids, Sterone, Ailanthusaltinin A, Ailanthaltone A

\section{Introduction}

Quassinoids, a group of bitter principles with various biological activities such as antimalarial, antitumor, antifeedant, anti-inflammatory, antiviral, and amoebicidal effects have attracted increasing interest in these years. ${ }^{1-4}$ Simaroubaceae plants are known to be producers of quassinoids and thus have been extensively studied. ${ }^{4}$ Ailanthus altissima Swingle, a plant in the family of Simaroubaceae, is indigenous to China and has been introduced to Europe and the United States in the 18th century. The bark of this plant is used as an astringent in traditional Chinese medicine for the treatment of diarrhea, bleeding and tinea. As a source plant of quassinoids, A. altissima was also widely investigated. Previous reports revealed the presence of
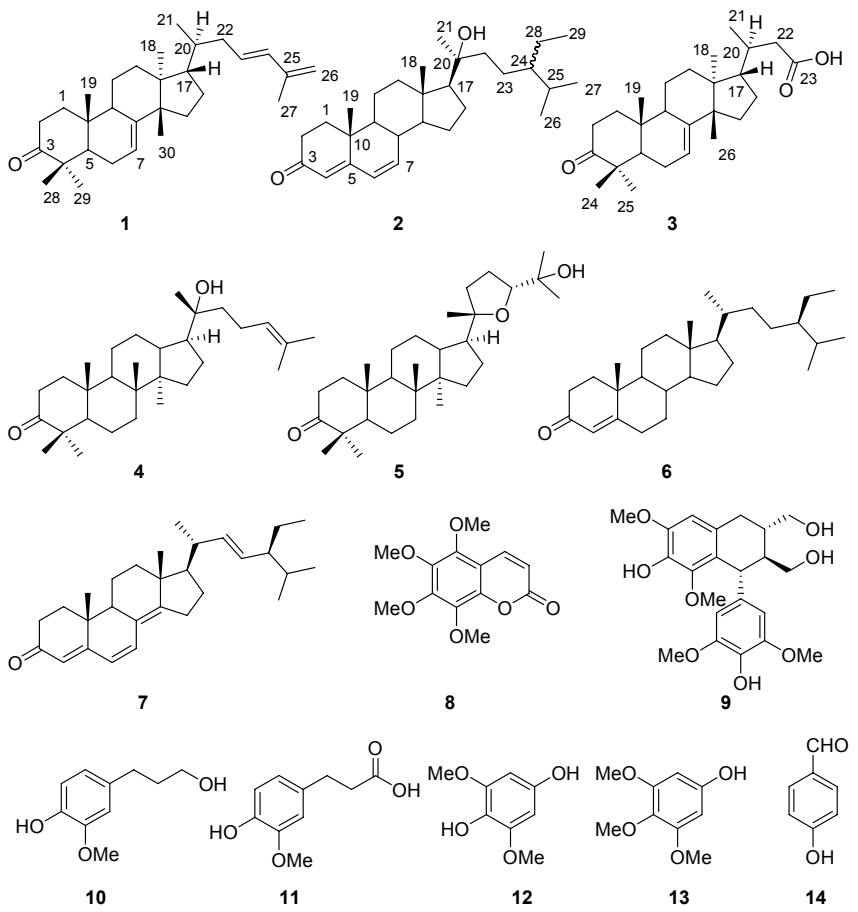

Figure 1. The structures of compounds 1-14. quassinoids, ${ }^{5-7}$ alkaloids, ${ }^{8-10}$ and phenolic constituents, ${ }^{11}$ and lipids in the plant. ${ }^{12}$ As a part of our study on medicinal plants, we investigated the stem bark of this plant, and 14 compounds (Figure 1) including a new tirucallane-type triterpenoid (1) and a new $\mathrm{C}_{29}$ sterone (2) were isolated. Herein, we describe the isolation and structure identification of the new compounds.

\section{Results and Discussion}

The molecular formula of compound 1 was determined to be $\mathrm{C}_{30} \mathrm{H}_{46} \mathrm{O}$ by its HRESIMS. The ${ }^{13} \mathrm{C}$ NMR and DEPT spectra exhibited signals for seven methyl (one secondary and six tertiary), nine methylene (one olefinic), seven methine (three olefinic), and seven quaternary carbons (two olefinic, one carbonyl). These data in combination with two olefinic carbon signals ( $\delta 117.8 \mathrm{CH}, 145.9 \mathrm{qC}$ ) suggested that $\mathbf{1}$ is a tirucallane-type triterpenoid containing the functionalities of a $\Delta^{7,8}$ double bond and a carbonyl group at C-3. Comparing its NMR data with those of 23,26-dihydroxytirucalla-7,24-dien-3-one ${ }^{13}$ suggested that they had the same framework. The difference was at the side chain linked to $\mathrm{C}-17 .{ }^{1} \mathrm{H},{ }^{1} \mathrm{H}$ COSY cross peaks of $\mathrm{H}-21 /$ H-20/H-22/H-23 ( $\delta 5.62) / \mathrm{H}-24(\delta 6.11)$ and HMBC correlations of H-27 $(\delta 1.83,3 \mathrm{H}, \mathrm{s}), \mathrm{H}-23, \mathrm{H}-24 / \mathrm{C}-25$ ( $\delta 142.2, \mathrm{qC})$; H-27/ $\mathrm{C}-26\left(\delta 114.0, \mathrm{CH}_{2}\right) ; \mathrm{H}-24 / \mathrm{C}-26, \mathrm{C}-27$ (Figure 2) established the $\Delta^{23,24}$ and $\Delta^{25,26}$ double bonds. The geometry of $\Delta^{23,24}$ was assigned as $E$-form by the large $J_{\mathrm{H}-23, \mathrm{H}-24}$ value of $15.5 \mathrm{~Hz}$. The relative configuration of $\mathbf{1}$ was determined by analysis of the NOESY spectrum, which showed the following correlations (Figure 3): H-2 $\beta / \mathrm{H}-28, \mathrm{H}-19, \mathrm{H}-9 / \mathrm{H}-18, \mathrm{H}-30 / \mathrm{H}-17$. Collectively, the structure of 1 was identified as $(13 \alpha, 14 \beta, 17 \alpha, 20 S, 23 E)$ -

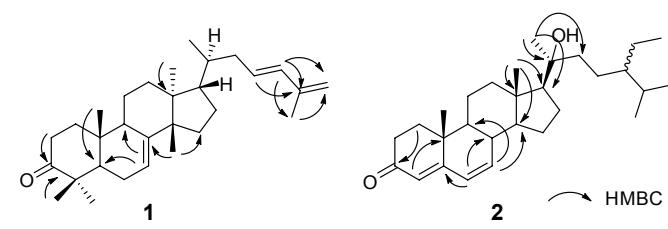

Figure 2. Important HMBC correlations for $\mathbf{1}$ and $\mathbf{2}$. 


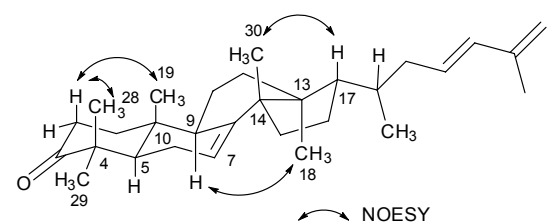

Figure 3. Important NOESY correlations for $\mathbf{1}$.

Table 1. NMR data for $\mathbf{1}^{a}$ and $\mathbf{3}^{a}$

\begin{tabular}{|c|c|c|c|c|}
\hline \multirow{2}{*}{ No } & \multicolumn{2}{|r|}{1} & \multicolumn{2}{|r|}{3} \\
\hline & ${ }^{13} \mathrm{C}^{b}$ & ${ }^{1} \mathrm{H}^{c}$ & ${ }^{13} \mathrm{C}^{b}$ & ${ }^{1} \mathrm{H}^{c}$ \\
\hline 1 & 38.5 & $\begin{array}{l}1.98(\mathrm{~m}) \\
1.45(\mathrm{~m})\end{array}$ & 38.4 & $\begin{array}{l}1.98(\mathrm{~m}) \\
1.45(\mathrm{~m})\end{array}$ \\
\hline 2 & 34.9 & $\begin{array}{l}2.74(\mathrm{dt}, 14.5,5.5) \\
2.22(\mathrm{~m})\end{array}$ & 34.9 & $\begin{array}{l}2.75(\mathrm{dt}, 14.5,5.5) \\
2.24(\mathrm{dt}, 14.5,3.5)\end{array}$ \\
\hline 3 & 216.9 & & 217.1 & \\
\hline 4 & 48.0 & & 47.8 & \\
\hline 5 & 52.3 & $1.70(\mathrm{~m})$ & 52.2 & $1.71(\mathrm{t}, 8.6)$ \\
\hline 6 & 24.3 & $\begin{array}{l}2.07(\mathrm{dt}, 16.2,3.3) \\
1.02(\mathrm{~m})\end{array}$ & 24.3 & $\begin{array}{l}2.08(\mathrm{ddd}, 16.2,8.6,3.1) \\
1.71(\mathrm{~m})\end{array}$ \\
\hline 7 & 117.8 & $5.29(\mathrm{dd}, 6.1,3.3)$ & 118.0 & $5.30(\mathrm{dd}, 6.1,3.1)$ \\
\hline 8 & 145.9 & & 145.6 & \\
\hline 9 & 48.4 & $2.27(\mathrm{~m})$ & 48.3 & $2.27(\mathrm{t}, 3.5)$ \\
\hline 10 & 34.9 & & 34.9 & \\
\hline 11 & 18.2 & $\begin{array}{l}1.55(\mathrm{~m}) \\
0.84(\mathrm{~m})\end{array}$ & 18.2 & $\begin{array}{l}1.58(\mathrm{~m}) \\
1.54(\mathrm{~m})\end{array}$ \\
\hline 12 & 33.4 & $\begin{array}{l}1.77(\mathrm{~m}) \\
1.63(\mathrm{~m})\end{array}$ & 33.4 & $\begin{array}{l}1.79(\mathrm{~m}) \\
1.65(\mathrm{~m})\end{array}$ \\
\hline 13 & 43.6 & & 43.6 & \\
\hline 14 & 51.5 & & 51.2 & \\
\hline 15 & 34.0 & $\begin{array}{l}1.50(\mathrm{~m}) \\
1.47(\mathrm{~m})\end{array}$ & 33.9 & $1.50(\mathrm{~m})$ \\
\hline 16 & 27.4 & $\begin{array}{l}1.95(\mathrm{~m}) \\
1.29(\mathrm{~m})\end{array}$ & 28.1 & $\begin{array}{l}1.94(\mathrm{~m}) \\
1.50(\mathrm{~m})\end{array}$ \\
\hline 17 & 52.8 & $1.46(\mathrm{~m})$ & 52.7 & $1.52(\mathrm{~m})$ \\
\hline 18 & 21.9 & $0.79(\mathrm{~s})$ & 21.9 & $1.45(\mathrm{~s}) 0.84(\mathrm{~s})$ \\
\hline 19 & 12.7 & $1.00(\mathrm{~s})$ & 12.7 & $1.50(\mathrm{~m}) 0.99(\mathrm{~s})$ \\
\hline 20 & 36.7 & $1.45(\mathrm{~m})$ & 33.9 & $\begin{array}{l}1.50(\mathrm{~m}) \\
1.93(\mathrm{~m})\end{array}$ \\
\hline 21 & 18.7 & $0.82(\mathrm{~d}, 5.7)$ & 19.2 & $0.97(\mathrm{~d}, 6.4)$ \\
\hline 22 & 39.5 & $2.25(\mathrm{~m}), 1.80(\mathrm{~m})$ & 41.3 & $\begin{array}{l}2.48(\mathrm{dd}, 3.1,147), \\
2.03(\mathrm{~m})\end{array}$ \\
\hline 23 & 129.5 & $\begin{array}{l}5.62 \\
(\mathrm{ddd}, 15.5,6.5,3.3)\end{array}$ & 179.7 & \\
\hline 24 & 134.0 & $6.11(\mathrm{~d}, 15.5)$ & 24.5 & $1.04(\mathrm{~s})$ \\
\hline 25 & 142.2 & & 21.6 & $1.11(\mathrm{~s})$ \\
\hline 26 & 114.0 & $4.85(\mathrm{~s})$ & 27.4 & $1.00(\mathrm{~s})$ \\
\hline 27 & 18.5 & $1.83(\mathrm{~s})$ & & \\
\hline 28 & 24.5 & $1.02(\mathrm{~s})$ & & \\
\hline 29 & 21.6 & $1.09(\mathrm{~s})$ & & \\
\hline 30 & 27.3 & $1.00(\mathrm{~s})$ & & \\
\hline
\end{tabular}

${ }^{a} \mathrm{In}_{\mathrm{CD}} \mathrm{Cl} ;{ }^{b}$ measured at $100 \mathrm{MHz} ;{ }^{c}$ measured at $400 \mathrm{MHz}$.

Lanosta-7,23,25-trien-3-one, and given a name ailanthusaltinin A.

Compound 2 had the molecular formula $\mathrm{C}_{29} \mathrm{H}_{46} \mathrm{O}_{2}$ deduced from its HRESIMS. The NMR data of $\mathbf{2}$ were similar to those of compound 6, except for the presence of the $\Delta^{6,7}$ double bond and a hydroxyl attached at C-20. ${ }^{1} \mathrm{H},{ }^{1} \mathrm{H}$ correlations of $\mathrm{H}-6(\delta$
6.09)/H-7 $(\delta 6.11) / \mathrm{H}-8(\delta 2.19) / \mathrm{H}-9(\delta 1.18)$ and HMBC correlations of H-6, H-18 ( $\delta$ 1.09)/C-5 ( $\delta$ 163.9), H-6/C-8, H-7/ C-8, C-9, C-14 suggested a $\Delta^{6,7}$ double bond (Figure 2 ). The chemical shift of C-20 $(\delta 75.2)$ and the molecular formula of 2 require a hydroxyl to be linked at C-20. The chemical shift for $\mathrm{H}_{3}-21$ at $\delta 1.26$ indicated the $20 S$-configuration ( $20 S: \delta 1.28$; 20R: $\delta 1.13) .{ }^{14,15}$ The absolute configuration at C-24 of 2 remained unresolved. Take these data together, the structure of compound 2 was elucidated as $(17 \alpha, 20 S)$-20-Hydroxy-stigmasta-4,6-dien-3-one, namely ailanthaltone A.

Known compounds were identified as (4,4,14-trimethyl-3oxo-24-nor-5 $\alpha, 13 \alpha, 14 \beta, 17 \alpha, 20 S$-chol-7-en-23-oic acid) (3), ${ }^{16}$ hydroxydammarenone I (4), ${ }^{17}$ ocotillone (5), ${ }^{18}$ stigmasta-4-ene3 -one (6), ${ }^{19}$ stigmasta-4,6,8(14), 22-tetraen-3-one (7), ${ }^{20}$ artelin (8), ${ }^{21}$ polystachyol (9), ${ }^{22}$ coniferyl alcohol (10), ferulic acid (11), 2,6-dimethoxyhydroquinone (12), antiarol (13), and 4-hydroxybenzaldehyde (14) by comparison with published literature data or direct identification by spectroscopic evidences. It was noted that compound $\mathbf{3}$ has been synthesized previously, ${ }^{16}$ however, it was isolated from natural sources for the first time. In addition, the NMR data of $\mathbf{3}$ were firstly assigned in this study (Table 1). Compounds 4, 5, and 9 were isolated from this plant for the first time. This study will help to get a deep insight into the chemical profiling of this medicinal plant.

\section{Experimental Section}

General Procedures. Optical rotations were recorded on a Horiba SEPA-300 polarimeter. UV spectra were measured on a Shimadzu UV-2401PC spectrophotometer. IR spectra were obtained on a Tensor 27 with $\mathrm{KBr}$ pellets. NMR spectra were recorded on a Bruker AM-400 or a DRX 500 or a Bruker Avance III-600 MHz spectrometer. ESI-MS and HRESIMS were determined with an API QSTAR Pulsar 1 spectrometer. Silica gel (200 - 300 mesh, 10 - $40 \mu \mathrm{m}$, Qingdao Marine Chemical Inc., Qingdao, China), RP-18 gel (40 - $63 \mu \mathrm{m}$, Daiso Co., Osaka, Japan), and Sephadex LH-20 (Amersham Biosciences, Uppsala, Sweden) were used for column chromatography.

Plant Material. The stem bark of $A$. altissima was purchased from Hunan Corporation of Materia Medica, Hunan Province, China, in July 2007, and authenticated by one of our authors (X. J. Zhou). A voucher specimen (CHYX-0084) was deposited at our laboratory in Kunming Institute of Botany.

Extraction and Isolation. The dried powdered stem bark of A. altissima $(10 \mathrm{~kg})$ was extracted with $\mathrm{EtOH}(2 \times 30 \mathrm{~L})$ to give an extract (720 g), which was suspended in water and partitioned by petroleum ether, EtOAc and $n$ - $\mathrm{BuOH}$ (each $3 \times 4 \mathrm{~L}$ ), respectively. The petroleum ether extracts $(150 \mathrm{~g})$ was divided into fractions A-D by a silica gel column eluted with petroleum ether with increasing amounts of EtOAc. Fr. C (19 g) was divided into frs. C-1-C-4 by a MCI gel CHP 20P column eluted with a gradient aqueous $\mathrm{MeOH}$. Compounds 4 (29 mg) and 5 (32 $\mathrm{mg}$ ) crystals were readily obtained from fr. C-2 (1.6 g) and fr. C-3 (2.1 g), respectively. The mother liquor of fr. C-3 (1.2 g) was separated by silica gel column $\left(\mathrm{CHCl}_{3} / \mathrm{EtOAC}, 100: 2\right)$, Sephadex $\mathrm{LH}-20\left(\mathrm{CHCl}_{3} / \mathrm{MeOH}, 6: 4\right)$ and finally preparative thin layer chromatography (PTLC) $\left(\mathrm{CHCl}_{3} / \mathrm{EtOAC}, 10: 0.3\right)$ to give 8 (132 mg). Fr. C-4 (1.6 g) was submitted to vaccum liquid 
Table 2. NMR data for $\mathbf{2}^{a}$

\begin{tabular}{|c|c|c|}
\hline \multirow{2}{*}{ No } & \multicolumn{2}{|r|}{2} \\
\hline & ${ }^{13} \mathrm{C}^{b}$ & ${ }^{1} \mathrm{H}^{c}$ \\
\hline 1 & 33.8 & $1.96(\mathrm{ddd}, 13.1,5.3,3.8) 1.67(\mathrm{~m})$ \\
\hline 2 & 33.8 & $\begin{array}{l}2.64(\mathrm{dt}, 17.7,5.3) \\
2.59(\mathrm{dt}, 17.7,3.8)\end{array}$ \\
\hline 3 & 199.7 & \\
\hline 4 & 123.5 & $6.66(\mathrm{~s})$ \\
\hline 5 & 163.9 & \\
\hline 6 & 127.9 & $6.09(\mathrm{dd}, 9.8,2.3)$ \\
\hline 7 & 141.4 & $6.11(\mathrm{dd}, 9.8,1.4)$ \\
\hline 8 & 37.0 & 2.19 (t-like, 10.6) \\
\hline 9 & 50.8 & $1.18(\mathrm{~m})$ \\
\hline 10 & 36.2 & \\
\hline 11 & 20.5 & $1.61(\mathrm{~m}), 1.45(\mathrm{~m})$ \\
\hline 12 & 40.1 & $2.11(\mathrm{dt}, 12.5,4.0), 1.22(\mathrm{~m})$ \\
\hline 13 & 43.9 & \\
\hline 14 & 53.7 & $1.21(\mathrm{~m})$ \\
\hline 15 & 24.1 & $1.24(\mathrm{~m}), 1.07(\mathrm{~m})$ \\
\hline 16 & 23.2 & $1.18(\mathrm{~m}), 1.08(\mathrm{~m})$ \\
\hline 17 & 57.3 & $1.21(\mathrm{~m})$ \\
\hline 18 & 13.5 & $0.92(\mathrm{~s})$ \\
\hline 19 & 16.2 & $1.09(\mathrm{~s})$ \\
\hline 20 & 75.2 & \\
\hline 21 & 26.1 & $1.26(\mathrm{~s})$ \\
\hline 22 & 42.5 & $1.44(\mathrm{~m}), 1.25(\mathrm{~m})$ \\
\hline 23 & 22.9 & $1.18(\mathrm{~m}), 1.08(\mathrm{~m})$ \\
\hline 24 & 46.2 & $0.91(\mathrm{~m})$ \\
\hline 25 & 29.0 & $1.66(\mathrm{~m})$ \\
\hline 26 & 19.2 & $0.81(\mathrm{~d}, 4.6)$ \\
\hline 27 & 19.5 & $0.81(\mathrm{~d}, 4.6)$ \\
\hline 28 & 22.3 & $1.81(\mathrm{~m}), 1.70(\mathrm{~m})$ \\
\hline 29 & 12.0 & $0.85(\mathrm{~d}, 7.4)$ \\
\hline
\end{tabular}

${ }^{a}$ In $\mathrm{CD}_{3} \mathrm{Cl} ;{ }^{b}$ measured at $100 \mathrm{MHz} ;{ }^{c}$ measured at $400 \mathrm{MHz}$.

chromatography (VLC) on a silica gel column eluted with petroleum ether/EtOAC (98:3) to afford one fraction (530 mg) which was passed through a Sephadex LH-20 column $\left(\mathrm{CHCl}_{3} /\right.$ $\mathrm{MeOH}, 6: 4)$ to afford $1(15 \mathrm{mg})$. Fr. B (8.5 g) was divided into B-1-B-3 over a silica gel column eluted with petroleum ether/ EtOAC (10:0.2). Fr. B-2 (1.8 g) was submitted to a Sephadex $\mathrm{LH}-20$ column $\left(\mathrm{CHCl}_{3} / \mathrm{MeOH}, 6: 4\right)$ and finally purified by PTLC $\left(\mathrm{CHCl}_{3} / \mathrm{EtOAc} / \mathrm{iPrOH}, 5: 1: 0.3\right)$ to yield 7 (10 mg). Fr. B-3 (5.5 g) was fractionated into B-3-1 and B-3-2 by a MCI gel CHP 20P column with 75\% aqueous MeOH. Fr. B-3-1 (400 $\mathrm{mg})$ was passed through Sephadex LH-20 $\left(\mathrm{CHCl}_{3} / \mathrm{MeOH}, 6: 4\right)$ followed by semi-preparative HPLC (Shimadzu, LC-10A HPLC system, Ultimate XB-C-18, $5 \mu \mathrm{m}, 10 \times 250 \mathrm{~mm}$, i.d., $92 \%$ aqueous $\mathrm{MeOH})$ to yield $2(6 \mathrm{mg})$ and $\mathbf{3}(72 \mathrm{mg})$. Fr. B-3-2 (1 g) was chromatographed on a RP-18 column ( $88 \%$ aqueous $\mathrm{MeOH})$ followed by PTLC $\left(\mathrm{CHCl}_{3} / \mathrm{EtOAc}, 10: 0.8\right)$ to give $6(173 \mathrm{mg})$.

The $n$-BuOH soluble extracts $(56 \mathrm{~g})$ were subjected to a MCI gel CHP 20P column eluted with gradient aqueous $\mathrm{MeOH}$ to give fractions E-I. Fr. G (3.4 g) was separated over a silica gel column (petroleum ether/ $\mathrm{Me}_{2} \mathrm{CO}, 5: 1$ ) to afford fractions $\mathrm{G}-1$ and G-2. Fr. G-2 (400 mg) was gel filtrated by Sephadex LH-20 $\left(\mathrm{CHCl}_{3} / \mathrm{MeOH}, 6: 4\right)$ and final semi-preparative HPLC (Shima- dzu, LC-10A HPLC system, Ultimate XB-C-18, $5 \mu \mathrm{m}, 10 \times$ $250 \mathrm{~mm}$, i.d., $67 \%$ aqueous $\mathrm{MeOH})$ to yield $9(9 \mathrm{mg})$ and $\mathbf{1 0}$ (15 mg). Fr. H (5.8 g) was divided into portions H1-H3 by VLC on silica gel with $\mathrm{CHCl}_{3} /$ EtOAc (6:1) as solvents. Fr. H-2 (800 $\mathrm{mg}$ ) was repeatedly purified by silica gel column (petroleum ether/ $\left.\mathrm{CHCl}_{3} / \mathrm{EtOAC}, 2: 4: 1\right)$ and final Sephadex LH-20 $\left(\mathrm{CHCl}_{3} /\right.$ $\mathrm{MeOH}, 6: 4)$ to afford $\mathbf{1 2}(18 \mathrm{mg}), \mathbf{1 3}$ (7 mg), and $\mathbf{1 4}(8 \mathrm{mg})$. Fr. H-3 (600 mg) was submitted to Sephadex LH-20 chromatography $(\mathrm{MeOH})$ followed by PTLC (petroleum ether/ $\mathrm{CHCl}_{3} /$ $\left.\mathrm{Me}_{2} \mathrm{CO}, 1: 5: 1\right)$ to give $11(20 \mathrm{mg})$.

$(13 \alpha, 14 \beta, 17 \alpha, 20 S, 23 E)$-Lanosta-7,23,25-trien-3-one (1): White solids; $R_{f}=0.65$, silica gel $G F_{254}, \mathrm{CHCl}_{3} / \mathrm{Me}_{2} \mathrm{CO}$ (10:0.4); $[\alpha]_{\mathrm{D}}^{18}-83.9$ (c 0.1, MeOH:CHCl $\left.\mathrm{CH}_{3} 1: 1\right)$; UV (MeOH): $\lambda_{\max }(\log$ $\varepsilon)=230.6$ (4.21); IR (KBr) $v_{\max } 2952,2933,1710,1630,1457$, $1370,965 \mathrm{~cm}^{-1} ;{ }^{1} \mathrm{H}(400 \mathrm{MHz})$ and ${ }^{13} \mathrm{C} \mathrm{NMR}(100 \mathrm{MHz})$ data: see Table 1; ESIMS (positive) $423\left[\mathrm{M}+\mathrm{H}^{+}, 445[\mathrm{M}+\mathrm{Na}]^{+}\right.$; HRESIMS (positive) $445.3447\left([\mathrm{M}+\mathrm{Na}]^{+}\right.$, calcd. for $\mathrm{C}_{30} \mathrm{H}_{46} \mathrm{ONa}$, 445.3446).

(17a,20S)-20-Hydroxy-stigmasta-4,6-dien-3-one (2): Colorless gums; $R_{f}=0.5$, silica gel $G F_{254}, \mathrm{CHCl}_{3} / \mathrm{Me}_{2} \mathrm{CO}(10: 0.5)$; $[\alpha]_{\mathrm{D}}^{24}-27.9(c 0.22, \mathrm{MeOH}) ; \mathrm{UV}(\mathrm{MeOH}): \lambda_{\max }(\log \varepsilon)=285.8$ (3.29). IR (KBr) $v_{\max } 3444,2957,2933,2869,1660,1616,878$, $804 \mathrm{~cm}^{-1}$; ${ }^{1} \mathrm{H}(400 \mathrm{MHz})$ and ${ }^{13} \mathrm{C}$ NMR $(100 \mathrm{MHz})$ data see Table 2; ESIMS (negative) $425[\mathrm{M}-\mathrm{H}]^{-}, 461[\mathrm{M}+\mathrm{Cl}]^{-}$; HRESIMS (positive) $449.3398\left([\mathrm{M}+\mathrm{Na}]^{+}\right.$, calcd. for $\mathrm{C}_{29} \mathrm{H}_{46} \mathrm{O}_{2} \mathrm{Na}$, 449.3395).

Acknowledgments. This work was financially supported by a Project from Scientific Research Fund of Hunan Provincial Education Department (no.08c626), a Key Project for Drug Innovation (2008ZX09401-004) from the Ministry of Science and Technology of China, "Talent Scholarship for the Youth of Yunnan" (No. 2007PY01-48), and "Xi-Bu-Zhi-Guang" Project from the Chinese Academy of Sciences.

Supporting Information. The ${ }^{1} \mathrm{H}$ and ${ }^{13} \mathrm{C}$ NMR spectra of compounds 1-3 are available on line.

\section{References}

1. Yan, X. H.;Cheng, J.; Di, Y. T.; Fang, X.; Dong, J. H.; Sang, P.; Wang, Y. H.; He, H. P.; Zhang, Z. K.; Hao, X. J. J. Agric. Food Chem. 2010, 58, 1572.

2. Beutler, J. A.; Kang, M. I.; Robert, F.; Clement, J. A.; Pelletier, J.; Colburn, N. H.; McKee, T. C.; Goncharova, E.; McMahon, J. B.; Henrich, C. J. J. Nat. Prod. 2009, 72, 503.

3. Lidert, Z.; Wing, K.; Polonsky, J.; Imakura, Y.; Okano, M.; Tani, S.; Lin, Y. M.; Kiyokawa, H.; Lee, K. H. J. Nat. Prod. 1987, 50, 442.

4. Guo, Z.; Vangapandu, S.; Sindelar, R. W.; Walker, L. A.; Sindelar, R. D. Curr. Med. Chem. 2005, 12, 173 .

5. Kubota, K.; Fukamiya, N.; Hamada, T.; Okano, M.; Tagahara, K.; Lee, K. H. J. Nat. Prod. 1996, 59, 683.

6. Tamura, S.; Fukamiya, N.; Okano, M.; Koyama, J.; Koike, K. Nat. Prod. Res. 2006, 20, 1211.

7. Tamura, S.; Fukamiya, N.; Okano, M.; Koyama, J.; Koike, K.; Tokuda, H.; Aoi, W.; Takayasu, J.; Kuchide, M.; Nishino, H. Chem. Pharm. Bull. 2003, 51, 385.

8. Zhang, L. P.; Wang, J. Y.; Wang, W.; Cui, Y. X.; Cheng, D. L. J. Asian Nat. Prod. Res. 2007, 9, 253.

9. Souleles, C.; Kokkalou, E. Planta Med. 1989, 55, 286.

10. Crespi-Perellino, N.; Guicciardi, A.; Malyszko, G.; Arlandini, E.; Ballabio, M.; Minghetti, A. J. Nat. Prod. 1986, 49, 1010. 
11. Hwang, S. W.; Lee, J. R.; Lee, J.; Kwon, H. S.; Yang, M. S.; Park, K. H. Heterocycles 2005, 65, 1963.

12. Feo, V. D.; Martino, L. D.; Quaranta, E.; Pizza, C. J. Agric. Food Chem. 2003, 51, 1177.

13. Luo, X. D.; Wu, S. H.; Ma, Y. B.; Wu, D. G. Phytochemistry 2000, $54,801$.

14. Nes, W. R.; Varkey, E.; Crump, D. R.; Gut, M. J. Org. Chem. 1976, 41,3429

15. Mijares, A.; Cargill, D. I.; Glasel, J. A.; Lieberman, S. J. Org. Chem. 1967, 32, 810.

16. Breen, G. J. W.; Ritchie, E.; Sidwell, W. T. L.; Taylor, W. C. Aust. J. Chem. 1966, 19, 455 .
17. Poejlans, B. L.; Cart, B. K.; Francis, T. A.; Hylandh, L. J.; Allaudeen, S.; Troupe, N. J. Nat. Prod. 1987, 50, 706.

18. Seger, C.; Pointinger, S.; Greger, H.; Hofer, O. Tetrahedron Lett. 2008, 49, 4313.

19. Georgesa, P.; Sylvestrea, M.; Ruegger, H.; Bourgeoisa, P. Steroids 2006, $71,647$.

20. Kobayashi, M.; Krishna, M. M.; Ishida, K.; Anjaneyulu, V. Chem. Pharm. Bull. 1992, 40, 72.

21. Brown, D.; Asplund, R. O.; McMahon, V. A. Phytochemistry 1975, 114, 1083.

22. Sadhu, S. K.; Phattanawasin, P.; Choudhuri, M. S. K.; Ohtsuki, T. M.; Ishibashi, M. J. Nat. Med. 2006, 60, 258. 